\title{
Conocimientos y experiencias de los estudiantes de enfermería sobre la leishmaniasis visceral durante la formación académica
}

Knowledge and experiences of nursing students about visceral leishmaniasis during their academic training

Saberes e vivências dos estudantes de enfermagem acerca da leishmaniose visceral durante a formação acadêmica

Ariane Moreira Coelho ${ }^{1}$, Raquel de Jesus Rocha da Silva ${ }^{2}$, Açucena de Farias Carneiro ${ }^{3}$, Alison Rener Araújo Dantas ${ }^{4}$, Daniele Pereira Soares ${ }^{5}$, Marcelo Costa Fernandes ${ }^{6}$

\section{Resumen:}

La Leishmaniosis Visceral, también conocida popularmente como calazar, es considerada una enfermedad contagiosa y un problema de salud pública presente en gran parte del territorio brasileño, y es el resultado de su innumerable propagación entre humanos y animales, de las complicaciones y de la probabilidad de letalidad que puede generar el no tratarla adecuadamente.

Objetivos: En este estudio se trató de analizar el discurso de los académicos de enfermería de una universidad pública del estado de Paraíba sobre el enfoque de la leishmaniasis visceral en el entorno de formación.

\footnotetext{
${ }^{1}$ Enfermeira formada pela Universidade Federal de Campina Grande, integrante do grupo de pesquisa Laboratório de Tecnologias de Informação e Comunicação em Saúde/LATICS/UFCG, Cajazeiras, Paraíba, Brasil. ORCID: 0000-0002-6815-1038, e-mail: coelhoariane1996@gmail.com;

${ }^{2}$ Acadêmica de enfermagem pela Universidade Federal de Campina Grande, integrante do grupo de pesquisa Laboratório de Tecnologias de Informação e Comunicação em Saúde/LATICS/UFCG, Cajazeiras, Paraíba, Brasil. ORCID: 0000-0002-9021-8593, e-mail: raquelrocha02@hotmail.com;

${ }^{3}$ Acadêmica de enfermagem pela Universidade Federal de Campina Grande, integrante do grupo de pesquisa Laboratório de Tecnologias de Informação e Comunicação em Saúde/LATICS/UFCG, Cajazeiras, Paraíba, Brasil. ORCID: 0000-0003-4832-9094, e-mail: fariasacucenna@gmail.com;

${ }^{4}$ Acadêmico de enfermagem pela Universidade Federal de Campina Grande, integrante do grupo de pesquisa Laboratório de Tecnologias de Informação e Comunicação em Saúde/LATICS/UFCG, Cajazeiras, Paraíba, Brasil. ORCID: 0000-0003-0182-1612, e-mail: renerdantas30@gmail.com;

${ }^{5}$ Enfermeira formada pela Universidade Federal de Campina Grande, Residente do programa de Residência Multiprofissional de Saúde da Família e Comunidade da Secretaria Municipal de Saúde de João Pessoa/Faculdade de Ciências Medicas da Paraíba, Brasil. ORCID: 0000-0001-8575-5880, e-mail: dani.b.d.d@hotmail. com;

${ }^{6}$ Enfermeiro, Doutor, Docente da Universidade Federal de Campina Grande, Líder do Grupo de Pesquisa Laboratório de Tecnologias de Informação e Comunicação em Saúde/LATICS/UFCG. Cajazeiras-PB-Brasil. ORCID: 0000-0003-1626-3043. Email: celo_cf@hotmail.com;
} 
Metodología: La metodología utilizada fue del tipo de estudio descriptivo, con un enfoque cualitativo, mediado por la investigación-acción que se refiere a los estudios sociales, con el objetivo de intervenir en la realidad, a través de acciones que buscan la solución de problemas colectivos, además del uso de artículos científicos sobre el tema.

Resultados: Se pudieron identificar los principales problemas y dificultades relacionados con el abordaje de la enfermedad durante el proceso formativo reportado por los alumnos, como el modelo tradicional de en señanza ampliamente utilizado, además de la deficiencia ante un bajo abordaje de la materia durante toda la graduación, obteniendo como consecuencia, su falta de preparación durante la aplicabilidad de la atención al actor social afectado por la enfermedad.

Consideraciones finales: Por lo tanto, fue posible desarrollar a lo largo de la investigación algunas actividades por medio de acciones educativas, con el objetivo de la sensibilización y el empoderamiento de los estudiantes frente a la enfermedad y el contexto en el que se inserta, además, se percibió la necesidad de nuevas investigaciones investigando en particular, como ha sido el aprendizaje de los estudiantes en otras instituciones de educación superior y sus Proyectos de Cursos Pedagógicos.

\section{Palabras clave:}

Leishmaniasis Visceral; Estudiantes de Enfermería; Conocimientos, Actitudes y Práctica en Salud.

\section{Abstract:}

Visceral Leishmaniasis, also populary known as calazar, is considered a contagious disease and a public health problem present in a large part of the Brazilian territory, and is the result of its innumerable spread among humans and animals, complications and the probability of let hality that failure otreat it properly cangenerate.

Objectives: This study sought to analyse the discourse of nursing academics from a public university in the state of Paraibaonthe approach to Visceral Leishmaniasis in the training environment.

Methodology: The methodology used was of the descriptive study type, with a qualitative approach, mediated by action-research that refers to social studies, aiming to intervene in reality, through actions that seek to solve collective problems, in addition to the use of scientific articles on the subject.

Results: It was possible to identify the main problems and difficulties related to the approach to the disease during the formative process reported by the students, such as the traditional teaching model widely used, in addition to the deficiency in the face of a low approach to the subject during the whole graduation, obtaining as a consequence, their unpreparedness during the applicability of the care to the social actor affected by the disease. 
Final considerations: Therefore, it was possible to develop throughout theres earch some activities by means of educational actions, aiming at the awareness and empowerment of students in face of the disease and the context in which it is inserted, in addition, it was perceived the need for new research investigating in particular, as has been the learning of students in other higher education institutions and their Pedagogical Course Projects.

\section{Keywords:}

Visceral Leishmaniasis; Nursing Students; Health Knowledge, Attitudes, Practice.

\section{Resumo:}

A Leishmaniose Visceral, também conhecida popularmente como calazar,é considerada uma doença contagiosa e um problema de saúde pública presente em grande tarde do território brasileiro, sendo resultado da sua inúmera disseminação entre seres humanos e animais, complicações e probabilidade de letalidade que o não tratamento adequado da mesma pode gerar.

Objetivos: $O$ presente estudo buscou analisar o discurso dos acadêmicos de enfermagem de uma universidade pública do estado da Paraíba sobre a abordagem da Leishmaniose Visceral no ambiente formativo.

Metodologia: A metodologia utilizada foi do tipo estudo descritivo, com abordagem qualitativa, mediado pela pesquisa-ação que se refere a estudos sociais, objetivando intervir na realidade, por meio de ações que buscam solucionar problemas coletivos, além da utilização de artigos científicos sobre o assunto.

Resultados: Foi possível identificar os principais problemas e dificuldades relacionados a abordagem da doença durante o processo formativo relatado pelos estudantes, tais como o modelo tradicional de ensino muito utilizado, além da deficiência frente a baixa abordagem sobre o assunto durante toda a graduação, obtendo como consequência, o despreparo dos mesmos durante a aplicabilidade do cuidado frente ao ator social acometido com a doença.

Considerações finais: Portanto, foi possível desenvolver ao longo da pesquisa algumas atividades por meio de ações educativas, objetivando a sensibilização e o empedramento dos estudantes frente a doença e o contexto na qual está inserida, além disso, percebeu-se a necessidade de novas pesquisas investigando em especial, como tem sido o aprendizado dos estudantes nas demais instituições de ensino superior e seus Projetos Pedagógicos de Cursos.

\section{Palavras chave:}

Leishmaniose Visceral;Estudantes de Enfermagem;Conhecimentos, Atitudes e Prática em Saúde. 


\section{Introdução}

A Leishmaniose Visceral (LV) é uma doença e problema de saúde pública presente em grande parte do território brasileiro, tomando proporções de caráter preocupante tanto pela sua inúmera disseminação entre seres humanos e animais, como também pelas diversas complicações e probabilidade de letalidade que o não tratamento precoce da mesma pode gerar.

A LV, também conhecida popularmente como calazar, é caracterizada como doença infecciosa, que costuma tomar proporções sistêmicas, crônica e não contagiosa. É causada pelo agente etiológico da classe dos protozoários e gênero Leishmania, pertencentes ao complexo Leishmania (Leishmania) donovani, acomentendo freqüentemente a África, Ásia, Europa e as Américas, sendo o complexo formado por três espécies de Leishmania, a L.(L.)donovani, L.(L.) infantume L.(L.)chagasi. No Brasi lo causador da leishmaniose visceral costuma ser a L. chagasi, espécie análoga a L. infantum, tendo como principal mecanismo de transmissão a picada do mosquito fêmea que se contaminou anteriormente em mamíferos infectado ${ }^{(1)}$.

Esse mosquito, considerado como principal meio de contaminação, é popularmente conhecido em todo território nacional como mosquito palha, birigui, tatuquiras entre outras denominações. Porém, ainda existem outras formas e mecanismos de transmissão a serem considerados, como a transmissão congênita que ocorre entre a mãe e o feto durante o período da gestação ou parto, transfusão sanguínea, acidentes laboratoriais e uso de drogas injetáveis com o compartilhamento de materiais infectados ${ }^{(2)}$.

Segundo o Sistema de Informação de Agravos de Notificação (SINAN), foram registrados no Brasil durante o ano de 2016, 3.200 casos de LV, totalizando uma incidência de 1,6 a cada 100.000 habitantes, havendo uma letalidade de $7,4 \%$ entre os acometidos, totalizando 265 óbi$\operatorname{tos}^{(3)}$

Observa-se, nesse contexto, a necessidade da formação permanente dos profissionais da saúde, em especial o enfermeiro já que acaba sendo o líder da equipe de saúde da família, para a identificação e elaboração de plano de cuidados para pessoas diagnosticadas com essa doença, com vistas a redução dos dados de morbimortalidade, bem como de medidas para evitar contrair a doença. Entende-se que os processos formativos devem começar já na graduação, uma vez que os mesmos podem reverberar positivamente na futura prática desses profissionais.

Portanto, diante do conteúdo apresentado anteriormente, indaga-se: qual os saberes e vivências dos estudantes de enfermagem acerca da leishmaniose visceral?

Logo, objetivou-se nesta investigação analisar o discurso dos acadêmicos de enfermagem sobre a abordagem da Leishmaniose Visceral no ambiente formativo.

\section{Metodologia}

Trata-se de estudo descritivo, com abordagem qualitativa, mediado pela pesquisa-ação. A pesquisa-ação, trabalha e refere-se a estudos sociais, objetivando intervir na realidade, por meio de ações que buscam solucionar problemas coletivos detectados previamente em uma determinada área, necessitando da participação 
cooperativa tanto dos pesquisadores, quanto dos atores sociais que se propuseram a participar da pesquisa ${ }^{(4)}$

A pesquisa foi realizada com 13 acadêmicos de enfermagem da Universidade Federal de Campina Grande (UFCG), situada na cidade de Cajazeiras, Paraíba, Brasil no mês de março de 2019. Reforça-se que a problemática debatida neste estudo emergiu a partir de inquietações dos próprios estudantes frente às lacunas vivenciadas durantes o processo formativo.

A coleta de dados se deu por meio de uma entrevista semiestruturada, ocorrendo de maneira individual e em local reservado, permitindo a exteriorização da opinião dos participantes. A ferramenta incluiu perguntas norteadoras discursivas, que favoreceu a liberdade de expressão dos participantes, a mesma foi gravada mediante a autorização prévia.

Durante a investigação foram estabelecidos alguns critérios de inclusão e exclusão, dessa maneira foram inclusos os alunos que se apresentarem regularmente matriculado no nono período e que estivessem cursando a disciplina do supervisionado II e como critérios de exclusão, os acadêmicos que tivessem participado de atividades extracurriculares de curso que apresentassem como foco a leishmaniose visceral.

Após a obtenção das entrevistas, foram realizadas algumas análises e estruturação das informações colhidas para o diagnóstico situacional, sendo produzidas mediante a utilização da técnica do Discurso do Sujeito Coletivo (DSC). Esse método, permite a construção de um pensamento coletivo, através do individual, associando discussões que apresentam semelhanças entre os diferentes depoimentos, possibilitando a condensação destas idéias para a formulação de um relato composto pela as idéias de um grupo $^{(5)}$
A produção o DSC, é necessário gerar Idéias Centrais (IC) e suas referentes Expressões-chaves $(\mathrm{ECH})^{(6)}$ Nesse contexto as IC representam as reproduções linguísticas, que apresentam o significado real dos discursos produzidos, já as $\mathrm{ECH}$ tratam-se de fragmentos do depoimento que revelam o principal conteúdo do discurso. Portanto o DSC é constituído pelas $\mathrm{ECH}$ que contém as IC iguais.

O percurso da pesquisa atendeu aos aspectos éticos preconizados pela Resolução 510/2016 do Conselho Nacional de Saúde, respeitando os princípios da autonomia, não-maleficência, beneficência, justiça e equidade ${ }^{(7)}$

A pesquisa teve início após a aprovação do projeto pelo Comitê de Ética em Pesquisa (CEP) da Universidade Federal de Campina Grande campus Cajazeiras, sob o parecer $\mathrm{n}^{\circ}$ 3.219.533. Foram assegurados o sigilo e o anonimato dos dados coletados e analisados, além das assinaturas dos termos de consentimento livre e esclarecido de maneira individual por cada participante.

\section{Resultados}

Ao relacionar e analisar alguns aspectos dos discursos colhidos após a realização da pesquisa, foi possível verificar o quanto os acadêmicos apresentam dificuldades e dúvidas sobre a LV, tanto nos conhecimentos científicos acerca da doença, como também sobre o acolhimento, tratamento, condutas e planos de cuidados necessários a serem prestados em tais situações. Dessa maneira, gerou-se três categorias a partir das análises citadas.

A primeira categoria trata-se da dificuldade dos acadêmicos frente à necessidade da elaboração de plano de cuidado voltado a um usuário 
que apresenta a LV dentro do seu ambiente de trabalho. Foi utilizado para a construção do DSC dessa categoria, o discurso de quatro acadêmicos.

Categoria 01. O despreparo por parte dos acadêmicos de enfermagem na construção do plano de cuidados ao usuário com Leishmaniose Visceral.

DCS01: Me pegou agora! Eu nunca pensei nisso! Não faço ideia! O plano de enfermagem no momento não... não me recordo. Iria estudar mais sobre a doença para tomar as medidas necessárias, não iria arriscar com o conhecimento que eu tenho hoje, que é pouco sobre a doença, eu acho que a maioria dos enfermeiros seriam pegos de surpresa na unidade, chega com isso pra eles, e... eu acho que eles iam pensar num plano B, estudar mais pra tomar todas as medidas corretas, eu jamais ia dá um de doido e fazer um plano de cuidado, umas coisas, que eu num... num soubesse o que estava fazendo, né?!

A segunda categoria aborda o questionamento sobre a baixa quantidade de vezes e oportunidade de contato com a abordagem do conteúdo teórico ainda dentro do ambiente de formação, tornando-se ineficaz a compreensão frente à doença em questão. Foi utilizado para a construção do DSC dessa categoria, o discurso de oito acadêmicos.

Categoria 02. Pontualidade na abordagem da temática ao longo do processo formativo.

DSC02: A gente teve contato com a explanação sobre essa patologia nas cadeiras iniciais do curso, e ficou uma coisa muito vaga, porque a gente só vê uma vez e não foi amplamente discutida ao longo do curso. Essa temática, principalmente
Artículo Investigación

esse tema, só foi abordado na disciplina de parasitologia humana, então realmente, ela deixa a desejar. A abordagem feita foi adequada o problema é que, é... a gente não teve mais contato, acabou, assim é uma coisa que acaba no esquecimento, é diferente de outras doenças que a gente vê durante o curso todo e de certa forma fica praticando, fica revisando, aquele, aquilo fica mais na cabeça da gente, do que uma disciplina que a gente só viu uma vez e que o conteúdo parou por ali, a gente não teve mais contato mais de nenhuma forma. A gente deveria ver mais ela na questão da clínica também, por que a gente tem os estágios, no supervisionado eram pra terem enfatizado mais a doença também.

A terceira categoria aborda como principal temática a colocação sobre o formato das aulas em que a doença foi trabalhada, trazendo como insatisfação a forma em que a mesma foi explanada e como a compreensão foi prejudicada devido ao modelo tradicional de ensino. Foi utilizado para a construção do DSC dessa categoria, o discurso de cinto acadêmicos.

Categoria 03. Educação bancária como modelo hegemônico na formação em enfermagem.

DSC03: Foi abordada a... só por exploração em sala de aula mesmo, aquela aula com apresentação de slides e a gente estudava em casa algumas coisas nos livros. Era abordada através de provas é... seminários. Era de uma forma bem... tradicional, no modo de ensino tradicional, é... o professor expo, expunha $o, o \ldots$ assunto e a gente estudava logo após. Assim o que eu achei de, de fragilidade é que devia sair um pouco daquela teoria, e agente praticar mais ou com caso clínico ou ... é... arranjar alguma metodologia pra gente praticar 
mais, pra fixar mais o assunto, porque só na teoria, acho que confunde muito, porque é uma doença que é muito semelhante com outras parasitose.

\section{Discussão}

Observa-se a partir do DSC01, o despreparo em relação a elaboração de plano de cuidado voltado a paciente com LV, circunstância alarmante, quando reflete-se sobre as imensuráveis perdas proveniente desta realidade, visto que este cenário traz prejuízo para a enfermagem quanto profissão.

Além disso, a população que necessita de cuidado também é atingida, sendo resultado, possivelmente, do modelo tradicional de ensino utilizado durante a formação, que não desenvolve o raciocínio crítico do aluno e consequentemente, não aperfeiçoa o conhecimento de maneira satisfatória.

A enfermagem costuma enfrentar alguns obstáculos na construção e consolidação de saberes, ligados à prática gerencial e principalmente assistencial, já que o desenvolvimento do processo de trabalho da enfermagem, trabalha justamente com a materialização da proposta de promover, preservar e/ou restabelecer a condição de saúde do cliente por meio da Sistematização da Assistência de Enfermagem (SAE), que aparece como ferramenta capaz de guiar e estruturar todo o complexo da assistência ${ }^{(8)}$ Por este motivo, juntamente com a precarização e deficiência do modelo de ensino, muitos acadêmicos costumam apresentar dificuldades no processo de ensino e prática do conhecimento adquirido.

Corroborando com essa ideia, para elaborar um plano de cuidado no contexto da LV, é ne- cessário antes de tudo, identificar as prioridades do usuário que apresenta a doença, para então construir e implementar uma assistência de enfermagem individualizada, sistemática, humanizada, acolhedora e pautada em embasamentos científicos ${ }^{(9)}$ Dessa maneira, o plano de cuidado pode ser realizado com base nos sinais e sintomas apresentados, como dor, alterações hepáticas, febre, entre outros sintomas.

Além disso, a LV ainda é altamente fatal em alguns países do continente africano, sendo resultado de tratamentos inadequados, incluindo a indisponibilidade de algumas drogas e o despreparo dos profissionais, levando assim ao crescimento nas taxas de resistência microbiana à alguns medicamentos, portanto, segundo o estudo, o diagnóstico preciso e o tratamento adequado realizado pelos profissionais é de extrema importância para a melhora dos resulta$\operatorname{dos}^{(10)}$

Foi possível identificar também que os participantes apresentam certo grau de deficiência em relação ao conhecimento sobre a doença propriamente dita, podendo ser justificada quando ressaltam a esporadicidade que a doença foi abordada ao longo do processo formativo de acordo com o DSC02.

A LV é grande desafio para a saúde pública do Brasil, já esta doença se configura com uma das enfermidades mais negligenciadas do mundo $^{(9)}$. Devido a isso, pode-se refletir sobre a relação desta realidade com o processo formativo dos graduandos e futuros profissionais, podendo considerar a possibilidade deste problema ser produto de uma má formação se tratando da LV. Cajazeiras, cidade do local desta pesquisa, encontra-se entre os sete municípios da Paraíba com maior número de casos de LV notificados nos períodos de 2007 a 2015, ressaltando, devi- 
do a estes dados epidemiológicos, a necessidade de tornar a LV uma temática mais habitual no processo formativo $^{(11)}$

Logo assim, os dados epidemiológicos mostram a necessidade de tornar a LV uma temática mais habitual no processo formativo, ou seja, é necessário que em outras etapas da graduação em enfermagem, seja reforçado o assunto como destaca o DCS02.

Visto isso e observando as discussões anteriores e suas consequências para a formação, foi possível compreender a necessidade de apresentar novas metodologias de ensino, como opções de desenvolver uma aprendizagem significativa, rompendo com o modelo tradicional de ensino mencionado pelo DSC03 e consequentemente com suas fragilidades.

Esse modelo tradicional de ensino citado pelo DSC03 também é conhecido como educação bancária, tratando-se de uma formação que torna o aluno um deposito de informações, tornando-se então, passivo ${ }^{(12)} \mathrm{Na}$ maioria das vezes este modelo de educação tem como resultado uma formação de sujeitos não pensantes, acomodados, que não questionam, consequentemente reféns e submissos de uma estrutura de poder.

Portanto, considerando suas consequências para a formação, é necessário apresentar outras metodologias de ensino, como opções de desenvolver uma aprendizagem significativa, rompendo com o modelo tradicional de ensino e consequentemente com suas fragilidades.

A formação dos acadêmicos do curso de enfermagem costuma limitar-se quase exclusivamente à modelos tradicionais de ensino como o modelo biomédico, prejudicando dessa maneira, a formação humana desses profissionais, deixando de lado as práticas cuidativas sensíveis e evidenciando uma deficiência humana e científica durante a assistência ${ }^{(13)}$

No contexto da LV, é possível utilizar inúmeras metodologias ativas durante a construção do conhecimento, facilitando a compreensão do conteúdo e levando a resolubilidade da problemática trazida pelo DSC03. O caso clínico, como um exemplo, mostra-se como uma importante alternativa, visto que o mesmo utiliza a simulação de casos reais, permitindo que os discentes mantenham contato com a realidade e consequentemente, preparando-se para a vida profissional, tornando-se uma alternativa frente à inquietação dentro do DSC01.

Deste modo, percebe-se que embora exista uma gama de métodos que favorecem para melhor formação, ainda se costuma optar com frequência pelo ensino tradicional como analisado dentro do DSC03, sendo possível refletir sobre a associação do déficit de conhecimento dos acadêmicos de enfermagem, com o modelo de ensino a qual os mesmos foram submetidos.

\section{Considerações finais}

Dessa maneira, foi possível analisar o discurso dos acadêmicos de enfermagem sobre a abordagem da LV no ambiente formativo, observando suas indagações e questionamentos ligados em especial ao formato tradicional de ensino, à limitada abordagem do conteúdo durante todo o processo de formação resultando em suas dificuldades durante a assistência ligada à temática dentro do ambiente de trabalho da assistência de enfermagem. 
Como desdobramento desta pesquisa, a partir do método de pesquisa ação, ocorreu a realização de ações educativas em conjunto pesquisador e pesquisados com a finalidade de trabalhar de maneira lúdica o compartilhamento de saberes envolta da LV com o intuito de sensibilizar, bem como empoderar os estudantes não somente compreender a doença, mas também todo o contexto social que a mesma está inserida e, desta forma, ser capaz de elaborar um plano de cuidado alinhado com a realidade do população sob sua realidade.

A investigação apresentou algumas limitações, estando entre elas a realização da pesquisa somente em uma instituição, dificultando a dis- cussão sobre o assunto de maneira generalizada. Acredita-se que se houvesse oportunidade de realizar outras pesquisas a respeito da LV em diferentes instituições públicas e particulares de ensino, os resultados seriam ainda mais satisfatórios, já que traria possivelmente, resultados mais precisos e completos.

Visto isso, faz-se necessário enfatizar sobre a necessidade de novos estudos ligados à temática nas demais instituições de ensino superior, investigando como tem sido trabalhada a doença no processo de formação e principalmente, como tem sido o aprendizado dos estudantes, analisando de maneira precisa seus conhecimentos, bem como tal temática se apresenta nos Projetos Pedagógicos de Curso. 


\section{Referências}

1. Neves DP. Parasitologia humana. $13^{\mathrm{a}}$ ed. São Paulo: Atheneu; 2016.

2. Brasil. Ministério da Saúde. Departamento de Vigilância Epidemiológica. Manual de vigilância e controle da leishmaniose visceral. Brasília: Ministério da Saúde; 2014.

3. Sistema de Informação de Agravos de Notificação -SINAN. Brasília: Leishmaniose: casos confirmados notificados no Sistema de Informação de Agravos de Notificação. 2016.Disponível em: http://portalms.saude.gov.br/saude-de-a-z/leishmaniose-visceral/11334-situacao-epidemiologica-dados Acesso em: 22 Set. 2020.

4. Thiollent M. Metodologia da pesquisa-ação. 18a ed. São Paulo: Cortez; 2011.

5. Lefevre F, Lefevre AMC. Discourse of the collective subject: social representations and communication interventions. Texto contexto enferm [Internet] 2014; 23(2): 502-7.

6. Lefévre F,Lefévre AMC. Depoimentos e discursos. Brasília:Liberlivro; 2005.

7. Brasil. Conselho Nacional de Saúde. Resolução no 510, de 07 de abril de 2016. Disponível em: http://conselho.saude.gov.br/ resolucoes/2016/Reso510.pdf. [Consulta: 22 Set. 2020]
8. Soares MI,ResckZMR, Terra FS, Camelo SHH. Sistematização da assistência de enfermagem: facilidades e desafios do enfermeiro na gerência da assistência. Esc Anna Nery 2015; 19(1): 47-53.

9. Neto VLS,Costa CS, Silva IKM, Negreiros NV, Godoy ECP, Silva BCO, et al. Perfil diagnóstico de enfermagem para pessoas com leishmaniose. RevEnfermCent-OesteMin 2017; 7:e1381.

10. Gidey K,Belay D, Hailu BY, Kassa TD, Niriayo YL. Visceral leishmaniasisy treatment out comeand associated factors in NorthernEthiopia. BioMedResearchInternational 2019.

11. Reis ABNS, Araújo ACC, Carvalho FMG, Gurgel GCDL. Perfil epidemiológico da leishmaniose visceral no Estado $\mathrm{Da} \mathrm{Pa}$ raíba no período de 2007 a 2015. In: 2do. Congresso Brasileiro de Ciências da Saúde. Anais. Campina Grande: Realize Eventos e Editora; 2017. p.1-12.

12. Freire P. Pedagogia da autonomia: saberes necessários à prática educativa. $43^{\mathrm{a}}$ ed. São Paulo: Paz e Terra; 2011.

13. Sobral FR,Campos CJG. Utilização de metodologia ativa no ensino e assistência de enfermagem na produção nacional: revisão integrativa. RevEscEnferm USP 2012; 46(1): 208-18.

Recibido: 17/07/20

Aceptado: 11/02/21 